\title{
Combined photoluminescence and Raman microscopy for the identification of modern pigments: explanatory examples on cross-sections from Russian avant-garde paintings
}

Alessia Artesani ${ }^{1 *} \mathbb{D}$, Marta Ghirardello ${ }^{1}$, Sara Mosca ${ }^{1,2}$, Austin Nevin ${ }^{3}$, Gianluca Valentini ${ }^{1}$ and Daniela Comelli ${ }^{1}$

\begin{abstract}
In conservation science, the identification of painting materials is fundamental for the study of artists' palettes, for dating and for understanding on-going degradation phenomena. For these purposes, the study of stratigraphic micro-samples provides unique information on the complex heterogeneity of the pictorial artworks. In this context, we propose a combined-microscopy approach based on the application of time-resolved photoluminescence (TRPL) micro-imaging and micro-Raman spectroscopy. The TRPL device is based on pulsed laser excitation (excitation wavelength $=355 \mathrm{~nm}, 1 \mathrm{~ns}$ pulse width) and time-gated detection, and it is suitable for the detection of photoluminescent emissions with lifetime from few nanoseconds to hundreds of microseconds. In this work, the technique is beneficially applied for identifying different luminescent semiconductor and mineral pigments, on the basis of their spectral and decay kinetic emission properties. The spatial heterogeneities, detected in the micro-sample, are investigated with Raman spectroscopy (785-nm in CW mode) for a further identification of the paint composition on basis of the molecular vibrations associated with the crystal structure. The effectiveness and limits of the proposed combined method is discussed through analysis of a corpus of stratigraphic micro-samples from Russian Avant-garde modern paintings. In the selected samples, the method allows the identification of modern inorganic pigments such as cadmium-based pigments, zinc white, titanium white, chrome yellow, ultramarine and cinnabar.
\end{abstract}

Keywords: Photoluminescence microscopy, Time-resolved photoluminescence, Raman microscopy, Painting materials, Pigment identification

\section{Introduction}

In conservation science, the identification of painting materials is fundamental for the study of artists' palettes, for dating and for understanding on-going degradation phenomena. The in-depth knowledge of artist materials plays a crucial role in the fine-tuning of restoration and conservation protocols. For these purposes, the study of

\footnotetext{
*Correspondence: alessia.artesani@polimi.it

1 Physics Department, Politecnico di Milano, Piazza Leonardo da Vinci 32, 20133 Milan, Italy

Full list of author information is available at the end of the article
}

stratigraphic micro-samples provides unique information on the complex heterogeneity of pictorial artworks.

In this contest, Raman spectroscopy is retained as standard non-destructive technique for pigment identification in polychrome artworks, as paintings, sculptures and ancient manuscripts [1-6]. The recent research has clearly demonstrated how the method can be highly effective both for the direct in situ analysis of artwork-with the aid of portable and compact devices [3] -and for the in-depth study of micro-samples in the laboratory [4-6]. The identification of materials is made even easier by online Raman spectra databases, which are accessible without any restrictions and usable 
also by non-expert users $[7,8]$. The main limit of the method remains the intrinsic weakness of the Raman scattering phenomenon, whose scattered signal can be hindered by other competitive processes, first of all the broadband fluorescence from some samples. One effective way to improve the efficiency of Raman spectroscopy is to combine several excitation wavelengths as reported in [6] in order to optimized the tradeoff between the signal to noise ratio and the competitive fluorescence baseline arising from different materials. Another possibility is the use of a single near-infrared laser, for reducing the fluorescent contribution, and applying specific acquisition protocols, such as the Subtracted Shifted Raman spectroscopy (SSRS) or the Shifted Excitation Raman Difference Spectroscopy (SERDS) [9-11]. All these strategies aim at diminishing the limitations imposed by fluorescent samples.

Similarly, UV-induced fluorescence microscopy, based on the use of conventional epi-fluorescence microscope coupled with filtered mercury lamp for sample excitation and with a color digital camera for image registration, is a widely employed method in restoration laboratories. However, the fluorescence phenomenon is considered only for qualitative inspection, allowing one to simply evaluate the distribution of heterogeneities on surfaces or on micro-samples [12]. This is due to the limited chemical specificity of the fluorescent processes, since-when considering organic compounds typically encountered in artworks as binders and varnishes - the emission occurs from a variety of fluorophores [13], giving rise to a broadband and often poorly specific signal.

Nonetheless, the investigation of the fluorescent or more generally photo-luminescent (PL) emission can be advantageous when investigating specific categories of highly luminescent pictorial materials. This is the case of luminescent semiconductor and mineral pigments (see Additional file 1: Table S1). In fact, in direct semiconductor pigments, the free pair electron-hole radiative recombination is related to the energy of the band gap and hence highly specific of the semiconductor type. In addition in both direct and indirect semiconductors a radiative recombination from trap states can occur, associated to the defects and impurities within the semiconductor crystal structure. This latter emission can be informative of the semiconductor material and in particular of its synthesis process [14-16]. Finally, in mineral pigments, the presence of substitutional ions can give rise to a specific PL emission. An illustrative example is the presence of $\mathrm{Cu}^{2+}$ ions in the cuprorivaite mineral, which give rise to the exceptional infrared emission of the Egyptian Blue pigment [17]. By employing a spectrally-resolved and time-resolved PL approach, the characteristics of the photoluminescence emissions, i.e. the emission spectrum and lifetime, can be retrieved providing key-parameters for the identification of luminescent pigments.

In this work, we investigate the combination of timeresolved photoluminescence (TRPL) microscopy and micro-Raman spectroscopy for pigment identification, taking the advantages of the different sensitivity of the two methods to the phenomena described before and of the elevated flexibility of both systems that are custombuilt. The effectiveness and limits of the proposed combined-analytical method are discussed through analysis of a corpus of stratigraphic micro-samples from precious Russian modern paintings, with a particular focus on semiconductor pigments-such as cadmium yellow/ orange, zinc white and titanium white-widely diffuse in the modern age. We considered here selected micro-samples from oil paintings by Mikjail Larionov (1881-1964) and Natalia Goncharova (1881-1962), leaders of the Russian avant-garde (also known as Rayionism), mainly active in the period between the 1912 and $1915[18,19]$.

\section{Materials and methods Materials}

Three micro-samples from Russian modern paintings are considered in this work, coming from Archivio Gallone hosted at the Physics Department at the Politecnico of Milan [20]. Samples were taken from two paintings belonging to a private collection and attributed one to Mikjail Larionov (following labelled as sample L5 and L6) and the other to Natalia Gocharova (sample G3). The Goncharova's painting belong to the 'Rayonist Forest' series, while Larionov's painting is untitled. Both paintings are not dated, but they supposedly painted after the 1914. Samples from these two paintings were prepared as stratigraphic cross-sections by embedding them in epoxy resin.

\section{Methods \\ Photoluminescence microscope}

The PL properties of micro-samples are probed with a TRPL microscope. A scheme and a detailed description of the setup is provided elsewhere [21] and here is briefly summarized. The system is based on a Q-switching laser source (FTSS 355-50, Crylas GmbH, Berlin, Germany, $\lambda=355 \mathrm{~nm}$, pulse energy $=70 \mu \mathrm{J}$, pulse duration $=1 \mathrm{~ns}$, repetition rate $=100 \mathrm{~Hz}$ ) and a fast time-gated intensified CCD camera, coupled together with an epi-fluorescence microscope. The microscope mounts a $15 \times$ and a $50 \times$ objectives, which allows analysis of a field of view of $900 \mu \mathrm{m}$ and $300 \mu \mathrm{m}$ in diameter, respectively, with a spatial resolution down to $1 \mu \mathrm{m}$ and $0.6 \mu \mathrm{m}$, respectively. The microscope is also equipped with 12 band-pass transmission filters (FKB-VIS-40, Thorlabs Inc, spectral 
range covered: $370-870 \mathrm{~nm}$ ) in the detection path. The time-gated camera allows the detection of a two multispectral imaging datasets of the PL emission occurring at nanosecond and microsecond timescales, as described in details in the "Protocol" section.

\section{Raman spectroscopy}

The Raman device is a flexible homemade system, described elsewhere [1]. The system is based on a solid state laser emitting at 785- $\mathrm{nm}$ in $\mathrm{CW}$ mode and on a spectrometer coupled to a cooled Si-based CCD camera. The detection of Raman peaks is made in the spectral range $130-3000 \mathrm{~cm}^{-1}$ with a spectral resolution close to $10 \mathrm{~cm}^{-1}$. The excitation and detection units are connected to a micro-probe that allows the detection of Raman spectra at high signal-to-noise-ratio on selected spots of $15 \mu \mathrm{m}$ in diameter at a working distance of around $2 \mathrm{~mm}$.

\section{Protocol}

The whole protocol is schematically represented in Fig. 1. The analysis starts with a first visual examination of the sample with a commercial epi-fluorescence microscope (Leica DM RE) equipped with two lamps (a tungsten one for observation under visible light and a mercury one for observation of the optical emission). The microscope is

\section{COMMERCIAL MICROSCOPE}

Visual examination under VIS and UV light for qualitative sample description

II. TIME-RESOLVED PHOTOLUMINESCENCE MICROSCOPY

Detection of multi-spectral images at nanosecond timescale for characterizing short living luminescence
Detection of multi-spectral images at microsecond timescale for characterizing long living luminescence

\begin{tabular}{|c|c|}
\hline$\nabla$ & $\nabla$ \\
\hline Images correction & or system efficiency \\
\hline$\nabla$ & $\boldsymbol{\nabla}$ \\
\hline $\begin{array}{l}\text { PL Mapping: } \\
\text { Images acquired in different spectral } \\
\text { range are merged in a False Colour } \\
\text { Image. Usually the combination is made } \\
\text { with red, blue and green (RGB) channels }\end{array}$ & $\begin{array}{l}\text { Spectrum Chart: } \\
\text { The Region Of Interest (ROI) is manually } \\
\text { selected. The average intensity of the PL } \\
\text { emission is reported versus the central } \\
\text { wavelengths of the filters }\end{array}$ \\
\hline & $\nabla$ \\
\hline & $\begin{array}{c}\text { Comparison of the spectrum with } \\
\text { literature or measurements on analytical } \\
\text { grades }\end{array}$ \\
\hline
\end{tabular}

III. MICRO-RAMAN SPECTROSCOPY

Map of samples heterogeneities with colour camera and Raman measurements on selected areas

Application of Subtracted Shifted Raman Spectroscopy (SSRS) protocol

Baseline subtraction

$\nabla$

Comparison of the spectrum with literature or measurements on analytical grades

Fig. 1 The measurement protocol 
coupled to a colour digital camera (NIKON D750). This provide a first evaluation of the complexity of the stratigraphy and of the colour of layers, helpful for a qualitative description of the sample.

The cross-section is then analyzed with the TRPL microscope system. A sequence of PL time-gated images at a fixed delay is recorded in different spectral bands. In the present case study, analysis of the emissions occurring at the nanosecond and microsecond timescales are achieved by employing, for the former, a gate with a temporal width of $\mathrm{w}=10 \mathrm{~ns}$ synchronous with laser pulse (delay $\mathrm{D}=0 \mathrm{~ns}$ ), whereas, for the latter, a gate with a temporal width of $\mathrm{w}=10 \mu \mathrm{s}$ set at a delay $\mathrm{D}=0.2 \mu \mathrm{s}$ after the pulsed excitation. Following correction for the spectral efficiency of the detector, this procedure gives rise to the creation of two time-gated multispectral imaging datasets, related to the spectral emission behavior of the cross-section in a certain temporal regime (nanosecond or microsecond) of the emission decay. Each dataset is composed of a sequence of grey-colour images related to the emission intensity of the sample in different spectral bands.

Following this, a subset of images of the multispectral dataset are merged, achieving in this way a false color image useful for material discrimination. The PL spectrum is further reconstructed selecting a region of interest (ROIs). In this reconstruction procedure, for the sake of simplicity, each bandpass filter is modelled as a Dirac delta function centered at the filter central wavelength and the spectral transmission of filters are accounted for in the overall spectral detection efficiency. The PL spectrum in each ROI is shown as the mean of intensity values within the ROI with error bars reporting the ROI standard deviation. Data obtained are compared with literature and standard samples purchased from Kremer Pigmente and Sigma Aldrich.

After micro-TRPL analysis, the cross-section is then investigated with Raman spectroscopy on analysis points selected on the basis of previous measurements and observations. Raman measurements are typically carried out with an acquisition time between 5 and $15 \mathrm{~s}$ and an irradiance on sample between 700 and $3500 \mathrm{~W} \mathrm{~cm} \mathrm{~cm}^{-2}$. On the basis of the collected and post-processed Raman spectra (baseline subtraction and SSRS), material identification is achieved through comparison with reference Raman data from a free online published database $[7,8]$ or with Raman spectra of standard samples purchased from Kremer Pigmente and Sigma Aldrich.

\section{Results}

Figure 2 displays the visible and UV-excited images of the three sample from Larionov and Goncharova paintings. The stratigraphy thickness is about $200 \mu \mathrm{m}$ and characterized by not well-defined layers. In fact, colours are one into the other, especially in samples L5 and G3, possibly as a consequence of the artists' style. In addition, the number of different colours included in these sample is limited: bright red and yellow are present in all samples, intense blue and dark green are mixed with the other colours, while the whites have defined boundaries with respect to the other layers. From the UV images additional details can be appreciated. In particular, within the yellow and orange layers in samples L5 and G3, bluish dots are visible, which increase the heterogeneity of these samples.

\section{Sample L5}

With a conventional microscope, it is possible to recognize three layers in the stratigraphic sample L5 (Fig. 1). From the top to the bottom, a first layer (Layer 1) is constituted by two shades of yellow paints (light one and dark one), mixed together with a greenish colour, that appears as stripes through the layer. Beneath, a white layer (Layer 2) mixed with brown is superimposed to a tiny blue layer (Layer 3), where blue pigments grains are coarsely mixed with a white paint. UV photography (Fig. 1) highlights the complex and heterogeneous morphology of the yellow layer, with the appearance of reddish and greenish emitting region and a relevant presence of luminescent heterogeneities in all the paint layers. The brown colour does not show any visible emission, and similarly the blue layer. The UV image does not underline any other specific detail.

TRPL microscopy is employed on sample L5 for the detection of the emission occurring at both the nanosecond and microsecond timescales, as described in the "Methods" section, giving rise to the reconstruction of the related multispectral datasets. As an example, in Fig. $3 \mathrm{a}$ it is shown the nanosecond time-gated dataset. On the nanosecond and microsecond timescale, the different spectral behaviour of the emission of each layer can be visualized by combining images acquired in different spectral bands in a false colour representation (Fig. 3b). In particular, the false colour image at the nanosecond and microsecond timescale of sample L5 shows the heterogeneous emission of the yellow layer that consist of a mixture of yellow paints. Further, in the blue layer it is possible to visualize the emission of some heterogeneities, invisible in standard microscopy in dark-field and epi-fluorescence mode.

The spectral profile of the luminescent pigments in the stratigraphy are reconstructing achieving the identification of three main luminescent pigments in sample L5. In details, the yellow layer (Layer 1) shows a short emission (lifetime of order of few ns) peaked in the spectral region between 450 and $500 \mathrm{~nm}$ and a microsecond emission in 


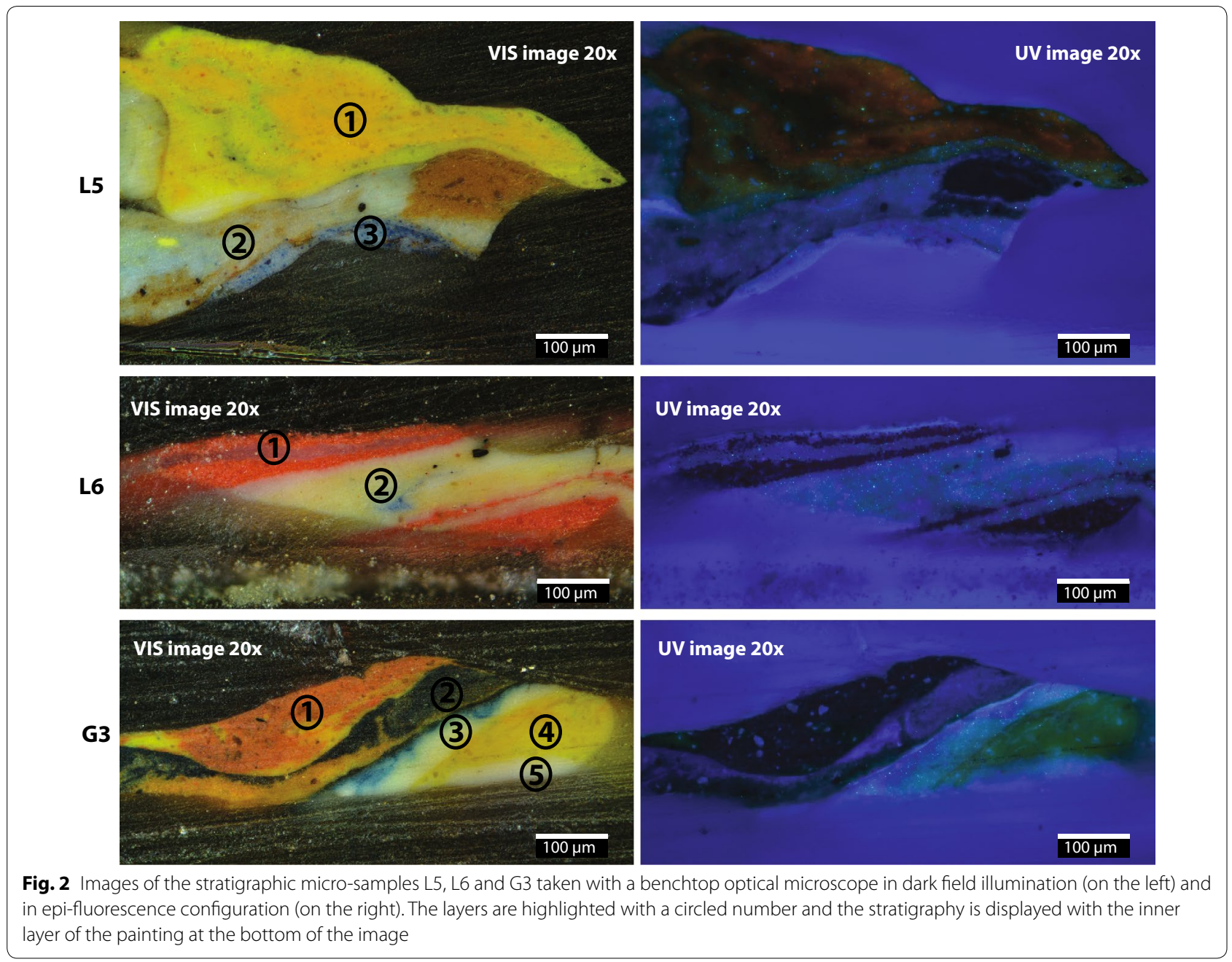

the NIR region, peaked around $700 \mathrm{~nm}$ (Fig. 3c). These features can be associated to the presence of cadmium yellow $\left(\mathrm{Cd}_{1-\mathrm{x}} \mathrm{Zn}_{\mathrm{x}} \mathrm{S}, 0<\mathrm{x}<0.25\right)$ paint, as largely discussed in $[22,23]$. The white layer (Layer 2) shows an intense blue short emission (380-400 nm) combined with a green microsecond emission (500-550 nm), properties that correspond to zinc white $(\mathrm{ZnO})$ paint $[15,24]$. As already quoted, PL micro-imaging reveals the presence of luminescent heterogeneities in the blue layer (Layer 3), characterized by microsecond NIR emission beyond $800 \mathrm{~nm}$, features that can be associated to trap state emission of cadmium based pigment, possibly cadmium red $\left(\mathrm{CdS}_{1-\mathrm{x}} \mathrm{Se}_{\mathrm{x}}, 0<\mathrm{x}<0.50\right)$ [25]. However, the reconstruction of the spectrum on nanosecond timescale (associated to the band edge emission of the semiconductor) is critical due to the very intense emission of $\mathrm{ZnO}$, diffusing within the stratigraphy of the micro-sample.

Complementary, the Raman analysis (Table 1) reveals the combined presence of lead chromate and blue phthalocyanine in the yellow layer (see Additional file 1), while cadmium Raman bands are not detected with the employed excitation. It is worth noticing that, on the basis of Raman vibrations, we can infer that the detected phthalocyanine pigment is a copper based phthalocyanine, largely used as blue synthetic dye. In the white layers, only Cinnabar Raman bands are detected. Finally, the blue layer presents the characteristic Raman peak of synthetic ultramarine blue. No other compounds are detected on the basis of Raman analysis (Fig. 4).

\section{Sample L6}

The second analysed sample is L6, from Larionov collection, which present a simpler stratigraphy than the other two samples. In the following, we report a synthesis of the obtained results. Referring to Fig. 2, bright red layers (Layer 1) occur at the top and at the bottom of a white layer (Layer 2). The central white body of the sample has in addition a yellowish tone and a blue residue. In a similar way to the previous sample, the main composition of the sample in terms of luminescent pigments is achieved 
(a)

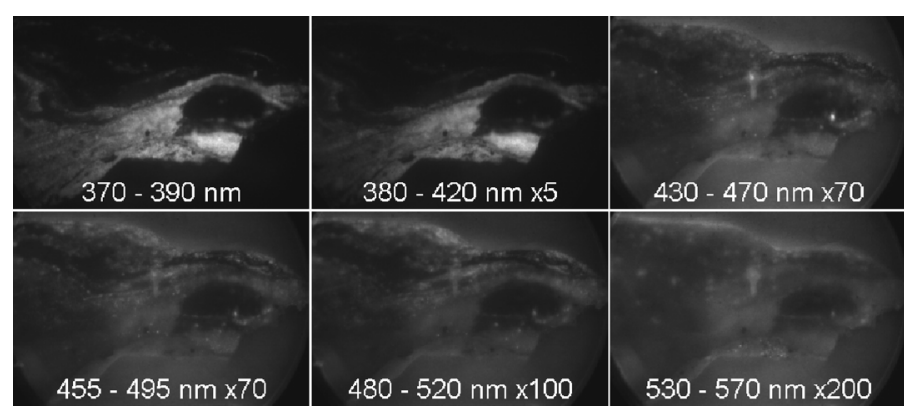

(b)

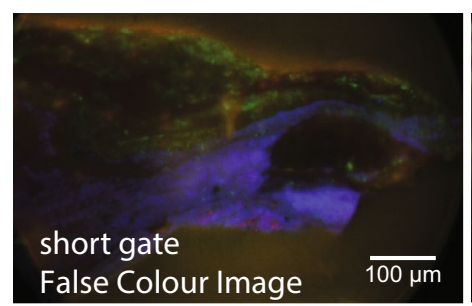

Time-gated dataset

\section{PL Images}

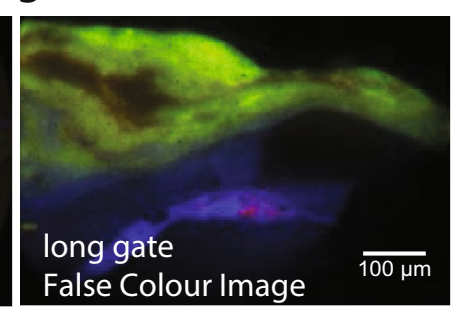

\section{(c)}
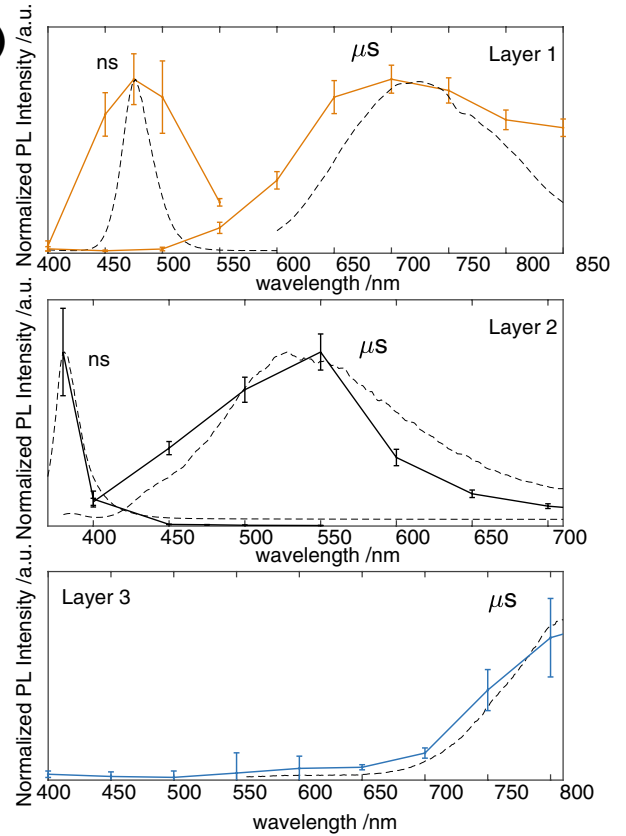

Fig. 3 a Spectral dataset of sample L5 acquired at the nanosecond timescale. In the label at the bottom of each image it is reported the analysed spectral band. b False colour images: ns timescale (blue $=370-390 \mathrm{~nm}$, green $=455-495 \mathrm{~nm}$ and red $=530-570 \mathrm{~nm}$ ) and microsecond timescale (blue $=530-570 \mathrm{~nm}$, green $=630-670 \mathrm{~nm}$ and red $=830-870 \mathrm{~nm}$ ). c Reconstructed spectra in selected ROIs of the yellow, white and blue layers at different timescale, compared with reference samples from Kremer Pigmente

Table 1 Pigments identified on sample L5 on the basis of Raman spectroscopy

\begin{tabular}{llll}
\hline Mineral name & Formula & Pigment name & Raman modes \\
\hline Lead chromate & $\mathrm{PbCrO}_{4}$ & Chrome yellow & 354(s), 375(m), 397(w), 839(vs) \\
- & $\mathrm{C}_{32} \mathrm{H}_{16} \mathrm{CuN}_{8}$ & Phthalocyanine blue & 256(m), 679(s), 746(s), 951(m) and \\
& & & in SM: 1143(m), 1337(s), 1448(m), \\
& & Vermillion & 253(s), 280(sh-W), 343(w) \\
Cinnabar & $\mathrm{HgS}$ & Ultramarine blue & 547(vs) \\
\hline
\end{tabular}

Italic: Wavenumber peak evidenced in the Raman spectra

through the TRPL micro-imaging analysis. As reported in Fig. 5, we detected an intense emission from the white layers, which occur at the nanosecond timescale in the spectral bands 380-400 $\mathrm{nm}$ and at the microsecond timescale in the green spectral bands $(500-550 \mathrm{~nm})$. This emission behaviour corresponds to the typical emission of the zinc white pigment [24]. Apart from this, we can observe that within the central white layer and on top of the red layer, there some luminescent dots, characterized by an intense emission around $500 \mathrm{~nm}$ at the nanosecond timescale and by an infrared emission $(700 \mathrm{~nm})$ in the microsecond gated window. This emission fingerprint is typical of Cd-yellow pigments. It is worth noticing that these luminescent dots within show some heterogeneities in terms of spectral emission at the nanosecond timescale, with dots emitting at $450 \mathrm{~nm}$ or $475 \mathrm{~nm}$ or $500 \mathrm{~nm}$ depending on their spatial position within the white layer, as is shown in Fig. 5c. This occurrence suggests that the yellow stripe within the white layer is made of a mixture of different cadmium yellows $\left(\mathrm{Cd}_{1-\mathrm{x}} \mathrm{Zn}_{\mathrm{x}} \mathrm{S}\right.$, $0<\mathrm{x}<0.25)$, and consequently displaying a NBE emission tuning from 450 to $500 \mathrm{~nm}[16,23,25]$. Going back to the paint stratigraphy, there is a tiny luminescent layer on top of it, which is not visible with conventional microscopy observations, but highly evident with the micro-TRPL analysis thanks to its specific emission at the nanosecond timescale centred at $630-670 \mathrm{~nm}$. In the case of this layer, we cannot uniquely associated this emission to a specific compound or pigment. A clearer distribution of the luminescent layers is finally obtained by merging PL images 

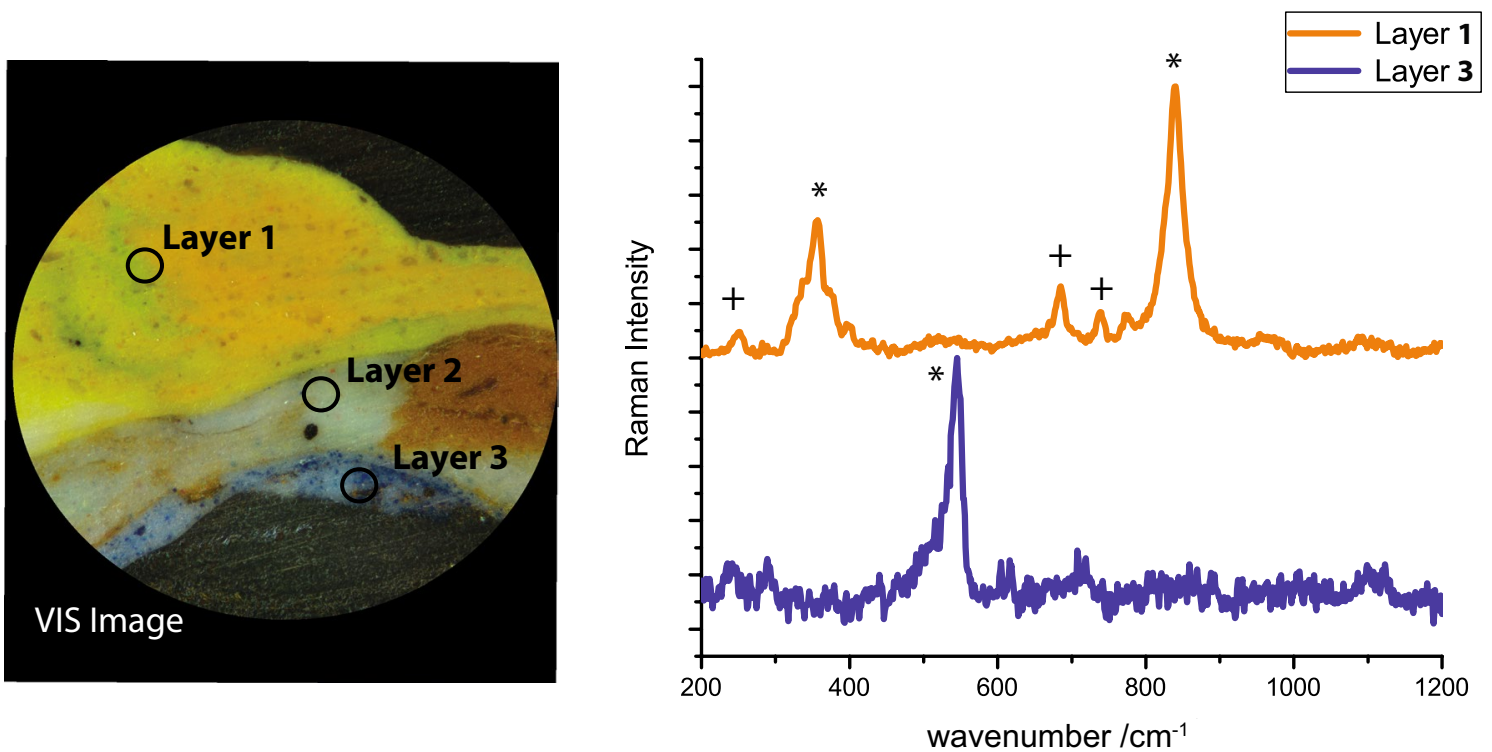

Fig. 4 Left panel: Analysis points investigated through Raman microscopy (highlighted as black circles over the colour picture of the sample). Right panel: Raman spectra of sample L5. Layer 1 shows the presence of Lead Chromate (354(s), 375(m), 397(w), 839(vs) $\mathrm{cm}^{-1}$ ) and Phthalocyanine Blue (256(m), 679(s), 746(s) cm $\mathrm{cm}^{-1}$ ) (see also Additional file 1). Layer 2 shows the Cinnabar peak at $250 \mathrm{~cm}^{-1}$. Layer 3 presents a peak at $548 \mathrm{~cm}^{-1}$, associated to Ultramarine Blue pigment

of the emission at the nanosecond timescale, as shown in Fig. 5b. Finally, we note that the red layers at the top and at the bottom of the stratigraphy L6 do not present any optical emission following UV excitation.

Results of micro-Raman spectroscopy are briefly summarized in Table 2. The method allows the identification of Cinnabar in the red layers, that was instead characterized by any PL emission. In the white layer, we detect Raman bands corresponding to different white components: the Hydrocerussite, Gypsum, and Zinc Oxide (Fig. 6). Because of the high sensitivity of Raman to the presence of Cinnabar a residue of this pigment is detected also in the white layer (Fig. 6). The outer layer, put in evidence by the PL analysis for its peculiar reddish emission, has a thickness of $10 \mu \mathrm{m}$, thus below the lateral resolution of our Raman system. Because of that, any material identification of this tiny layer was not possible.

\section{Sample G3}

Goncharova cross-section G3 is not made by a clear stratigraphy. Colours are one into the others in accordance with the painting technique of the artist. White, yellow, orange-yellow and dark green are recognizable as main colours. Specifically, Layer 1 and Layer 2 are mainly composed of dark yellow and orange pigments; the latter layer includes also a dark green stripe. Layer 3 and Layer 5 are white, the first one with blue inclusion coming from a thin layer between Layer 2 and 3. A bright yellow pigment (Layer 4) divides the two white layers. Epi-fluorescence microscopy does not add any details with respect to the visible microscopic image.

The TRPL micro-imaging measurements reveal that there are only two luminescent layers in this stratigraphy, Layer 3 and 5. The PL emission at the nanosecond timescale let us to clearly identify the use of zinc white as the white pigment in both the layers (Fig. 7). As shown in previous samples, its presence can be easily detected thanks to its typical emission below $400 \mathrm{~nm}$ ascribed to the direct electron-hole recombination and to the broad green emission associated to trap states. Beside this, Layer 3 has an additional emission occurring at the microsecond timescale and peaked in the near-infrared region, unexpected for the zinc white pigment. Such an infrared emission is instead peculiar of the rutile polymorph of $\mathrm{TiO}_{2}$ [26], used as a modern white pigment from the middle of twentieth century.

Raman spectroscopy is performed to confirm such a hypothesis. The results (Table 3 ) show that, as expected, the two white layers are characterized by different vibrational modes. First, in Layer 5 is confirmed the presence of zinc white as main pigment. No other components are detected in this layer. On reverse, Layer 3 has the typical fingerprint of Anatase, superimposed to the zinc white peak, as shown in Fig. 8. Finally, Raman spectroscopy recognizes Lead Chromate in the yellow layer (Layer 4), which instead has no luminescent emission. 


\section{Conventional Microscope}

(a)

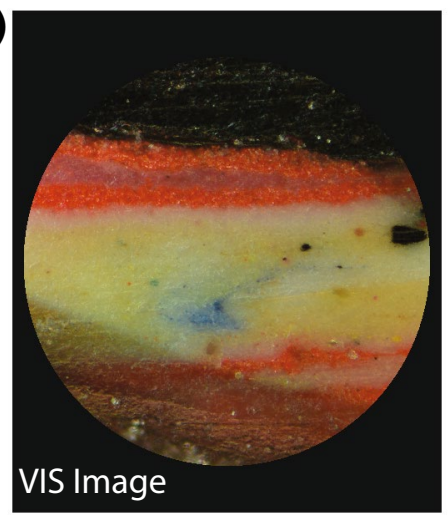

(b)

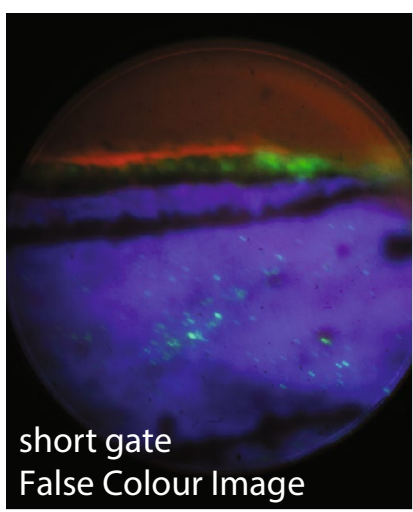

TRPL Microscopy

(c)

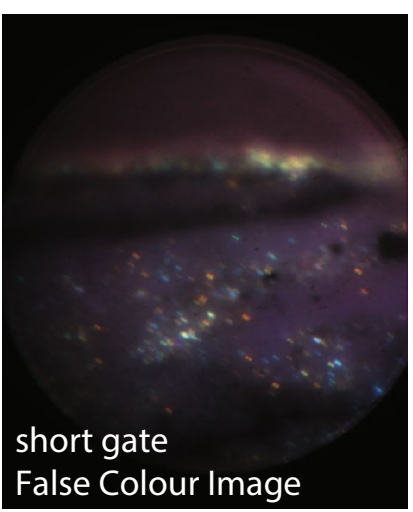

\section{Spectral Analysis}

(d)
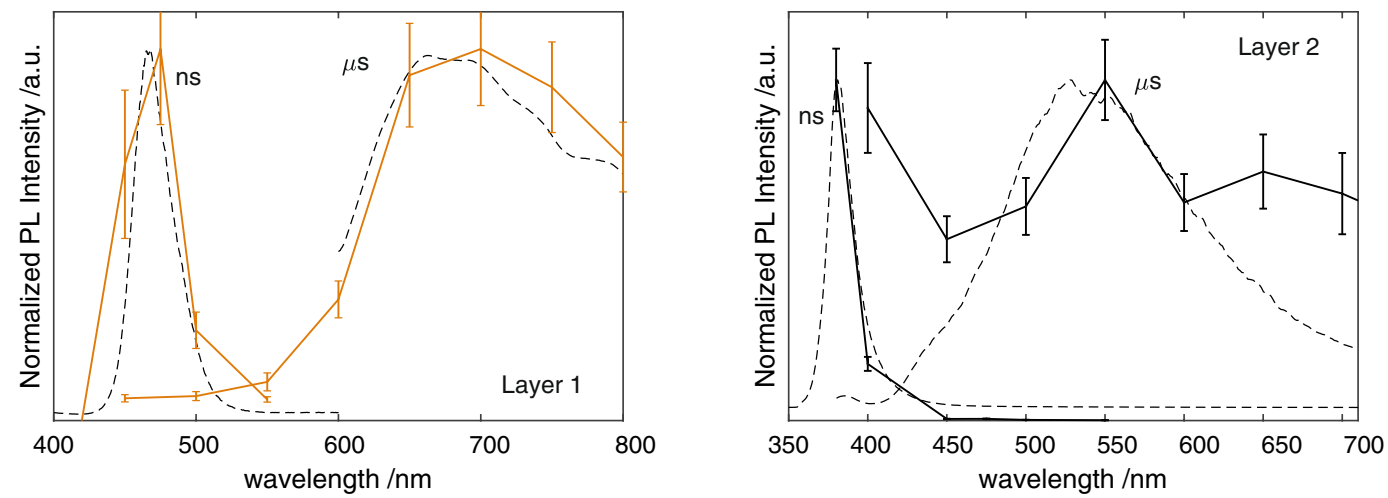

Fig. 5 a Detail of sample L6 investigated with TRPL microscopy. b False colour image at the nanosecond timescale (blue channel $=370-390 \mathrm{~nm}$, green channel $=480-530 \mathrm{~nm}$ and red channel $=630-670 \mathrm{~nm}$ ). c False colour image at the nanosecond timescale where blue $=430-470 \mathrm{~nm}$, green $=445-495 \mathrm{~nm}$ and red $=480-530 \mathrm{~nm}$. $\mathbf{d}$ Reconstructed spectra in selected ROIs of the Layer 1 (red) and Layer 2 (white) at different timescale, identified as cadmium based pigment $\left(\mathrm{Cd}_{1-x} \mathrm{Zn}_{x} \mathrm{~S}\right)$ and zinc white pigment $(\mathrm{ZnO})$

Table 2 Pigments identified on sample L6 on the basis of Raman spectroscopy

\begin{tabular}{|c|c|c|c|}
\hline Mineral name & Formula & Pigment name & Raman modes $\left(\mathrm{cm}^{-1}\right)$ \\
\hline Cinnabar & $\mathrm{HgS}$ & Vermillion & $253(\mathrm{~s}), 280(\mathrm{sh}-\mathrm{w}), 343(\mathrm{w})$ \\
\hline Hydrocerussite & $\mathrm{Pb}_{3}\left(\mathrm{CO}_{3}\right)_{2}(\mathrm{OH})_{2}$ & Lead white & $1051(\mathrm{vs}), 1384(\mathrm{w})$ \\
\hline Calcium sulphate dihydrate & $\mathrm{CaSO}_{4} \cdot 2 \mathrm{H}_{2} \mathrm{O}$ & Gypsum & $\begin{array}{l}419(\mathrm{~m}), 490(\mathrm{w}), 619(\mathrm{w}), \\
669(\mathrm{w}), 1008(\mathrm{vs})\end{array}$ \\
\hline Zinc oxide & $\mathrm{ZnO}$ & Zinc white & $331(w), 383(w), 438(s)$ \\
\hline
\end{tabular}

Italic: Wavenumber peak evidenced in the Raman spectra

\section{Discussion}

The results of the pigments identification are summarised in Table 1. PL approach recognizes $\mathrm{ZnO}$ as the main white pigment in all samples, while the presence of $\mathrm{TiO}_{2}$ is found in G3 sample. The yellow and orange pigments have been associated to cadmium-based pigments. Raman measurements complete the list and in Larionov samples allowed the detection of Cinnabar $(\mathrm{HgS})$ as red colour (which instead has any luminescent emission when excited with UV). In the yellow layer of L4 sample, the use chrome yellow (or lead chromate) can be recognized, while the greenish stripes within the yellow layer is due to the presence of the blue Phthalocyanine dye. Two white pigments required a further discussion. The first 

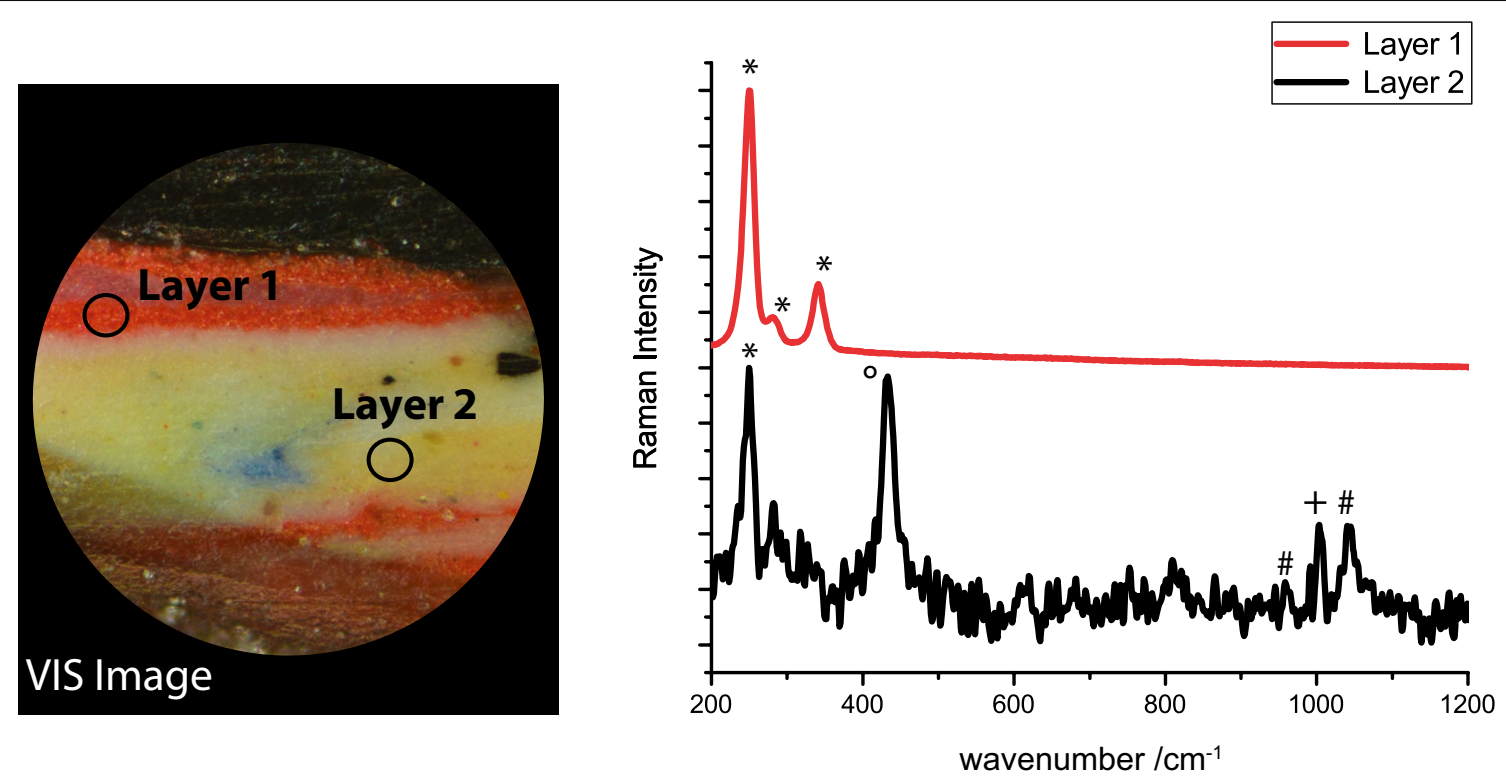

Fig. 6 Left panel: Analysis points investigated through Raman microscopy (highlighted as black circles over the colour picture of the sample). Right panel: Raman spectra of the samples L6. Layer 1 shows the presence of Cinnabar (250(vs), 280(sh-w) and 343(m) $\left.\mathrm{cm}^{-1}\right)$. Layer 2 shows the peak of Hydrocerussite $\left(1050 \mathrm{~cm}^{-1}\right)$, Gypsum $\left(1000 \mathrm{~cm}^{-1}\right)$ and Zinc Oxide $\left(438 \mathrm{~cm}^{-1}\right)$. In addition, a residue of Cinnabar $\left(250(\mathrm{~s}) \mathrm{cm}^{-1}\right)$ is detected in Layer 2

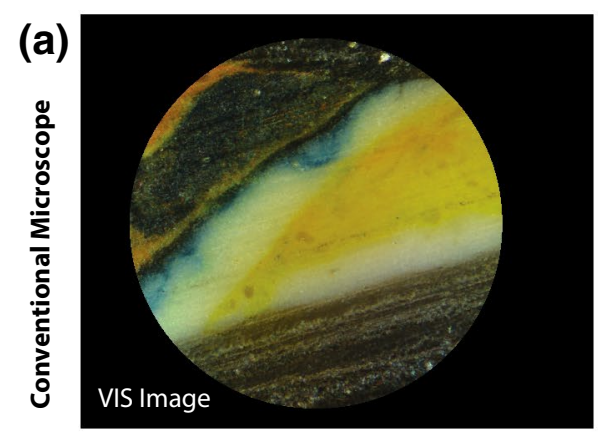

(b)
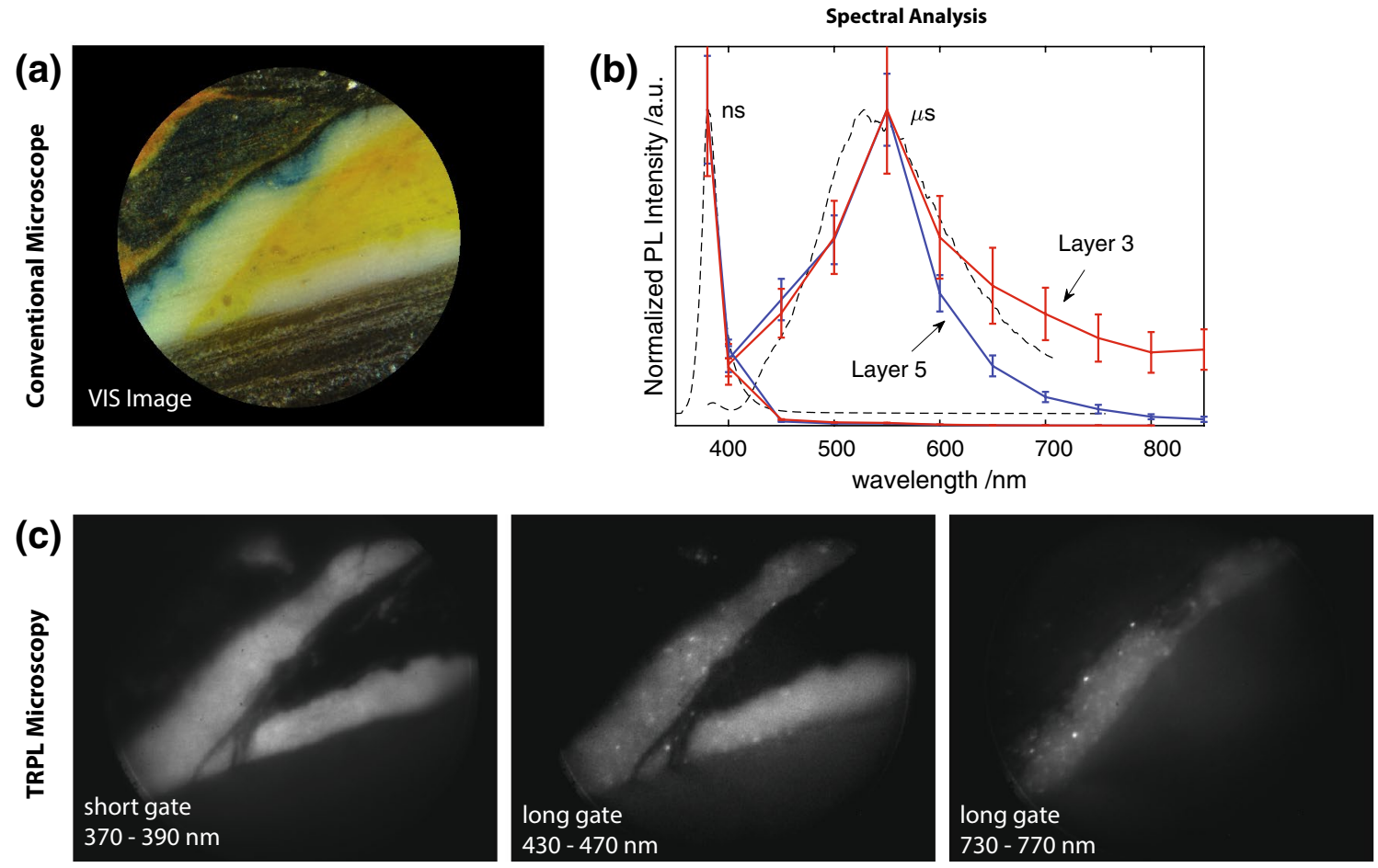

Fig. 7 a Detail of sample G3 investigated with PL microscopy. b Reconstructed spectra in selected ROls of the Layer 3 and Layer 5 (the two white), identified as zinc white pigment with traces of titanium white in Layer 3. c False colour images: nanosecond timescale gated image (370-390 nm) and microsecond timescale gated image (BP450/40 on the left, BP750/40 on the right) 
Table 3 Pigments identified on sample G3 on the basis of Raman spectroscopy

\begin{tabular}{llll}
\hline Mineral name & Formula & Pigment name & Raman modes $\left(\mathbf{c m}^{\mathbf{- 1}}\right)$ \\
\hline Zinc oxide & $\mathrm{ZnO}$ & Zinc white & $331(\mathrm{w}), 383(\mathrm{w})$ 438(s) \\
Anatase & $\mathrm{TiO}_{2}$ & Titanium white & $147(\mathrm{vs}), 198(\mathrm{vw}), 396(\mathrm{~m}), 515(\mathrm{~m})$ 640(m) \\
Lead chromate & $\mathrm{PbCrO}_{4}$ & Chrome yellow & $354(\mathrm{~s}), 375(\mathrm{~m}), 397(\mathrm{w}), 839(\mathrm{vs})$ \\
\hline
\end{tabular}

Italic: Wavenumber peak evidenced in the Raman spectra
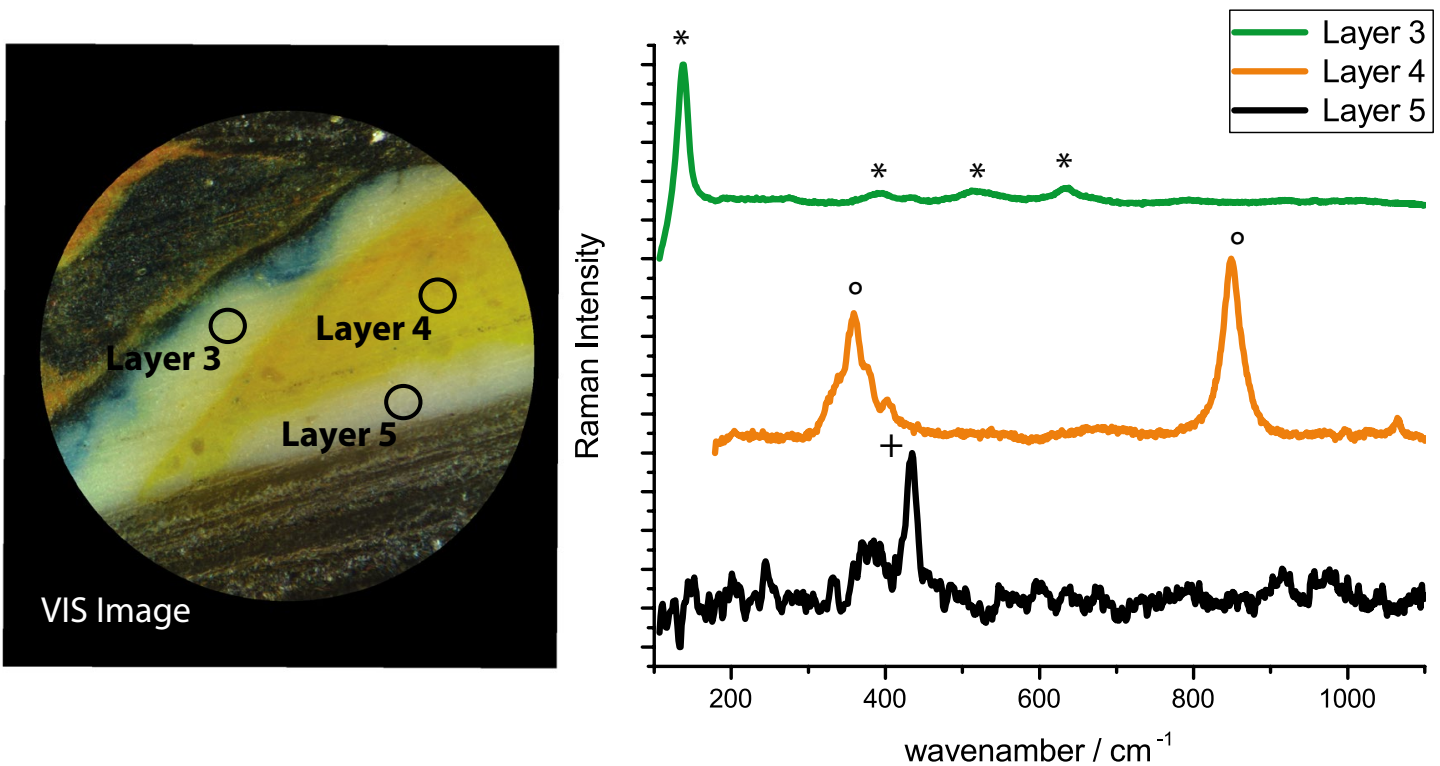

Fig. 8 Left panel: Analysis points investigated through Raman microscopy (highlighted as black circles over the colour picture of the sample). Right panel: Raman spectra of the samples G3. Layer 3 shows the presence of Anatase (147(vs), 198(vw), 396, $515,640 \mathrm{~cm}^{-1}$ ). Layer 5 is composed by Zinc Oxide (332(w), 383(w) 438(s) $\mathrm{cm}^{-1}$ ), whereas Layer 4 is made of Lead Chromate (354(s), 375(m), 397(w), 839(vs) cm ${ }^{-1}$ )

one is lead white in sample L6. This white pigment has two crystalline structures (cerussite and hydrocerussite) and it highly absorbs above $5 \mathrm{eV}$ [27]. It has been shown that when excited below this threshold (as in our case, around $3.5 \mathrm{eV}$ ), both crystal structure show an emission in the green due to trap states. In case of sample L6, this emission is superimposed to the green and more intense band of the zinc white pigment. Thus, its presence is hardly detectable with the use of PL techniques when it is mixed with other strongly luminescent pigments. On reverse, Raman spectroscopy has clearly inferred its presence. A second observation is related to titanium white in G3 sample. In this case, Raman spectroscopy allowed to detect the presence of the Anatase form of $\mathrm{TiO}_{2}$, whereas TRPL analysis it is possible to suppose the presence of the Rutile form of $\mathrm{TiO}_{2}$. Indeed, the two crystal forms are usually present in titanium white pigments, especially in the early production of the pigment [26]. From an historical point of view, this result indicates that the particular micro-sample analyzed might belong to a series of Goncharova of her Paris period, since titanium white is expected in oil painting after the 1920, when a French firm started an intense production [28, 29]. As final observation, the use of these pigments has been detected in other works belonging to the Russian avant-gard, as reported in already published literature [30-32].

The results of the case studies presented in this work put in evidence the sensitivity of TRPL and Raman spectroscopies to different materials. We have illustrated how in case of artist materials, the complementarity of these two methods can be highly advantageous: the timeresolved PL microscopy is highly sensitive to the presence of luminescent semiconductor pigments. Further, it gives an insight on the distribution of the luminescent compounds and on the material heterogeneity, whereas Raman spectroscopy can confirm and/or complement the information through a vibrational pattern specific of the chemical composition. The use of micro-Raman spectroscopy alone does not allow the identification of all the pigments present in a complex stratigraphy: indeed, as illustrated in the present case, modern semiconductor pigments are not or are hardly detectable with Raman 
microscopy, even when employing a proper post-processing data (like SSRS). Similarly and complementary, TRPL microscopy has allowed only the identification of luminescent pigments and cannot add any information on materials that do not have a specific luminescent fingerprint. Instead, when considered together, the two methods have provided a clearer view of the complexity of the paints and pigment mixture employed by the two Russian artists, as deeply discussed above.

The high-complementarity of Raman and PL spectroscopy has already been exploited in the past [33-35]. The great advantage of combining these two techniques is that they are both optical ones. Indeed, many efforts have been devoted to develop hybrid setups based on a common pulsed laser source and capable of in situ recording Raman and time-resolved photoluminescence spectra from the same analysis point with applications relevant to the geoscience [36] and conservation science field $[37,38]$. With the same idea in mind, in the future it could be possible to develop an hybrid microscope, with exchangeable laser excitation, dichroic and transmission filters, capable of combining Raman and TRPL measurements in a unique set-up (Table 4).

A comparison of the proposed approach with other more-conventional approaches/techniques applied to the analysis of paint stratigraphy is finally provided and summarized in Table 5. Scanning Electron Microscopy coupled to Energy-Dispersive X-Ray (SEM-EDX) spectroscopy allows one to infer the elemental composition of paint layers with a lateral resolution close to hundreds of nanometres. The method is often combined with microAttenuated Total Reflectance (ATR-FTIR) spectroscopy for detecting and identifying organic and inorganic compounds [6]. The two methods are highly complementary and display a high material sensitivity, nonetheless their main experimental limit is that they can require specific sample treatments and thus cannot be retained completely non-invasive. In fact, for SEM-EDX analysis the sample surface needs to be metallized and subsequent sample investigations require a gentle polishing action that inevitably would remove the first micrometres of the sample surface. In case of ATR-FTIR analysis, the measurement requires the contact between the diamond probing head of the system and the surface of the sample and this contact may induce mechanical damages (indentations) at the sample surface, especially in presence of soft materials as paints.

In this context, our approach-based on Raman and TRPL studies-add further information to the ones achieved with the conventional approach based on SEM-EDX and ATR-FTIR, providing clues on the presence of materials with specific Raman or PL

Table 4 List of pigments found in the Russian avant-garde painting cross-sections [30]

\begin{tabular}{|c|c|c|c|c|c|}
\hline Colour & Pigment & On the market & Sample & $\begin{array}{l}\text { Identification provided } \\
\text { by Raman }\end{array}$ & $\begin{array}{l}\text { Identification } \\
\text { provided } \\
\text { by TRPL }\end{array}$ \\
\hline \multirow[t]{4}{*}{ White } & Lead white & Antiquity & L6 & $x$ & - \\
\hline & Gypsum & Antiquity & L6 & $x$ & - \\
\hline & Zinc white & 1834 & L5, L6, G3 & $x$ & $x$ \\
\hline & Titanium white & 1940 & G3 & $x$ & $x$ \\
\hline \multirow[t]{4}{*}{ Yellow-red } & Vermillion & Antiquity & L5, L6 & $x$ & - \\
\hline & Chrome yellow & 1814 & $L 5, G 3$ & $x$ & - \\
\hline & Cadmium red & 1921 & L6 & - & $x$ \\
\hline & Cadmium yellow & 1840 & L5, L6 & - & $x$ \\
\hline \multirow[t]{2}{*}{ Blue } & Ultramarine & 1828 & L5 & $x$ & - \\
\hline & Phthalocyanine & 1936 & L5 & $x$ & - \\
\hline
\end{tabular}

Table 5 Principal conventional techniques applied to the analysis of paint stratigraphy

\begin{tabular}{llll}
\hline Technique & Identifies & Lateral resolution & Sample preparation \\
\hline SEM-EDX & Elements $(Z>10)$ & $\sim 100 \mathrm{~nm}$ & Metallization \\
ATR-FTIR & Organic and Inorganic compounds & $1-10 \mu \mathrm{m}$ & None \\
Raman & Organic and Inorganic compounds & $1-10 \mu \mathrm{m}$ & None cleaning \\
Micro-TRPL & Luminescent compounds & $1-10 \mu \mathrm{m}$ & None ${ }^{\mathrm{a}}$ \\
\hline
\end{tabular}

The main material identification, lateral resolution, sample preparation requirements and possible damages associated to the measurements are reported

a Raman and PL applications do not cause any damages to the sample as long as the powder density is kept far below the damage threshold [39] 
fingerprints. Moreover, being non-contact and noninvasive the proposed approach can be considered as mandatory in presence of high-valuable samples, for which any surface pre-treatment is allowed, or for the rapid and non-invasive screening of a large number of samples. In a future research, it will be of interest to study-on the same paint stratigraphy-how many pigments can be identified with the combination of TRPL and Raman measurements with respect to the combination of SEM-EDX and ATR-FTIR or ATR-FTIR and Raman.

\section{Conclusion}

In this work, we have applied two techniques, based on TRPL micro-imaging and on micro-Raman spectroscopy, that have been rarely used together as microscopy methods. We propose here this novel approach for the investigation of pigments and artist materials. The method instead of being alternative to other conventional analysis protocol (as the previously quoted SEM-EDX and ATR-FTIR analyses) provides complementary data for further understanding the complex and heterogeneous material composition of paint layers in paintings. In the future, taking into account the high sensitivity of the two methods to the detection of minerals, the proposed approach could be extended to other case studies, as stratigraphic micro-samples taken from ancient sculpture, wall painting and heritage stones.

\section{Additional file}

Additional file 1: Table S1. List of the main semiconductor and mineral pigments that can be found in modern paintings. For every pigment is reported (i) the general name, (ii) the chemical formula and the crystal structure, (iii) the band gap energy in eV (and the corresponding wavelength/nm) known from literature. (iv) and (v) report the near-band-edge (NBE) and trap state (TS) wavelength emission under near UV excitation, respectively. (vi) the origin of trap state emission as found in literature. Figure S1. Raman spectrum of Layer 1 in sample $L 5$ detected with the grating $600 \mathrm{lpmm}$ in the spectral range $200-2000 \mathrm{~cm}^{-1}$. It shows the presence Lead chromate (354(s-br), 375(sh-m), 839(vs)) and Phthalocyanine Blue. Blue numbers highlights the Raman bands used for the identification of Phthalocyanine blue (256(m), 679(s), 746(s) $951(\mathrm{~m}), 1143(\mathrm{~m}), 1337(\mathrm{~s})$, 1448(m), 1523(vs))

\section{Authors' contributions}

All authors contributed to research. AA and MG were responsible for photoluminescence measurements, analyses. SM carried out Raman spectroscopy measurements and analyses. DC coordinated the publication. All authors were responsible for interpretation of results. All authors read and approved the final manuscript.

\section{Author details}

1 Physics Department, Politecnico di Milano, Piazza Leonardo da Vinci 32, 20133 Milan, Italy. ${ }^{2}$ Central Laser Facility (CLF), Research Complex at Harwell, STFC Rutherford Appleton Laboratory, Harwell Campus, Didcot OX11 0QX, UK. ${ }^{3}$ Istituto di Fotonica e Nanotecnologie - Consiglio Nazionale delle Ricerche (IFN-CNR), Piazza Leonardo da Vinci, 20133 Milan, Italy.

\section{Acknowledgements}

Not applicable.

\section{Competing interests}

The authors declare that they have no competing interests.

Availability of data and materials

Please contact author for data requests.

Funding

Not applicable.

\section{Publisher's Note}

Springer Nature remains neutral with regard to jurisdictional claims in published maps and institutional affiliations.

Received: 4 November 2018 Accepted: 2 March 2019

Published online: 19 March 2019

\section{References}

1. Mosca S, et al. Identification of pigments in different layers of illuminated manuscripts by X-ray fluorescence mapping and Raman spectroscopy. Microchem J. 2016;124:775-84.

2. Casadio F, Daher C, Bellot-Gurlet L. Raman spectroscopy of cultural heritage materials: overview of applications and new frontiers in instrumentation, sampling modalities, and data processing. Top Curr Chem. 2016;374(5):62. https://doi.org/10.1007/s41061-016-0061-z.

3. Colomban P. The on-site/remote Raman analysis with mobile instruments: a review of drawbacks and success in cultural heritage studies and other associated fields. J Raman Spectrosc. 2012;43(11):1529-35.

4. Doulgeridis M, Burgio L, Anglos D, Melessanaki K, Clark RJH. Pigment identification in paintings employing laser induced breakdown spectroscopy and Raman microscopy. Spectrochim Acta Part B At Spectrosc. 2002;56(6):905-13.

5. Smith GD, Clark RJH. Raman microscopy in art history and conservation science. Stud Conserv. 2014;46(sup 1):92-106.

6. Lauwers D, Hutado AG, Tanevska V, Moens L, Bersani D, Vandenabeele P. Characterisation of a portable Raman spectrometer for in situ analysis of art objects. Spectrochim Acta Part A Mol Biomol Spectrosc 2014;118:294-301.

7. Ruff. Database of Raman spectroscopy, X-ray diffraction and chemistry of minerals. 2012. http://rruff.info/. Accessed 03 Feb 2019.

8. IRUG. IRUG spectral database. 2016. http://www.irug.org/search-spect ral-database. Accessed 03 Feb 2019

9. Rosi F, et al. Study of Raman scattering and luminescence properties of orchil dye for its nondestructive identification on artworks. J Raman Spectrosc. 2013;44(10):1451-6.

10. Bell SEJ, Bourguignon ESO, Dennis A. Analysis of luminescent samples using subtracted shifted Raman spectroscopy. Analyst. 1998;123(8):1729-34.

11. Conti C, Botteon A, Bertasa M, Colombo C, Realini M, Sali D. Portable sequentially shifted excitation Raman spectroscopy as an innovative tool for: in situ chemical interrogation of painted surfaces. Analyst. 2016;141(15):4599-607.

12. Sandu ICA, Schäfer S, Magrini D, Bracci S, Roque CA. Cross-section and staining-based techniques for investigating organic materials in painted and polychrome works of art: a review. Microsc Microanal. 2012;18(4):860-75.

13. Nevin A, Comelli D, Valentini G, Cubeddu R. Total synchronous fluorescence spectroscopy combined with multivariate analysis: method for 
the classification of selected resins, oils, and protein-based media used in paintings. Anal Chem. 2009;81(5):1784-91.

14. Bellei $\mathrm{S}$, et al. Multianalytical study of historical luminescent lithopone for the detection of impurities and trace metal ions. Anal Chem. 2015;87(12):6049-56.

15. Artesani A, et al. Photoluminescence properties of zinc white: an insight into its emission mechanisms through the study of historical artist materials. Appl Phys A Mater Sci Process. 2016;122(12):1053.

16. Ghirardello $\mathrm{M}$, et al. Time-resolved photoluminescence microscopy combined with X-ray analyses and Raman spectroscopy sheds light on the imperfect synthesis of historical cadmium pigments. Anal Chem. 2018;90(18):10771-9.

17. Accorsi $G$, et al. The exceptional near-infrared luminescence properties of cuprorivaite (Egyptian blue). Chem Commun. 2009;23:3392-4.

18. Dabrowski M. The formation and development of rayonism. Art J. 1975;34(3):200-7.

19. Parton A. Russian 'Rayism', the work and theory of Mikhail Larionov and Natalya Goncharova 1912-1914: Ouspensky's four-dimensional super race? Leonardo. 2006;16(4):298.

20. Gallone. Archivio Gallone|Dipartimento di Fisica - Politecnico di Milano. https://www.fisi.polimi.it/it/servizi/archivio_gallone. Accessed 03 Feb 2019.

21. Comelli D, et al. Time-resolved photoluminescence microscopy for the analysis of semiconductor-based paint layers. Materials. 2017;10(11):1335.

22. Comelli D, MacLennan D, Ghirardello M, Phenix A, Patterson CS, Khanjian H, Gross M, Valentini G, Trentelman K, Nevin A. Degradation of cadmium yellow paint: new evidence from photoluminescence studies of trap states in Picasso's Femme (Époque des "Demoiselles d'Avignon"). Anal Chem. 2019;91(5):3421-8. https://doi.org/10.1021/acs.analchem.8b04914.

23. Cesaratto A, et al. Analysis of cadmium-based pigments with timeresolved photoluminescence. Anal Methods. 2014;6(1):130-8.

24. Comelli $D$, et al. On the photoluminescence changes induced by ageing processes on zinc white paints. Microchem J. 2018;139:467-74.

25. Rosi F, et al. UV-Vis-NIR and microRaman spectroscopies for investigating the composition of ternary $\mathrm{CdS}_{1-x} \mathrm{Se}_{x}$ solid solutions employed as artists' pigments. Microchem J. 2015;125:279-89.

26. Mosca S, et al. New insights into the complex photoluminescence behaviour of titanium white pigments. Dye Pigment. 2018;155:14-22.
27. Gonzalez V, Gourier D, Calligaro T, Toussaint K, Wallez G, Menu M. Revealing the origin and history of lead-white pigments by their photoluminescence properties. Anal Chem. 2017;89(5):2909-18.

28. McCrone WC, Fitzhugh EW. Artists' pigments: a handbook of their history and characteristics, Vol. 3. J Am Inst Conserv. 2006;38(3):483.

29. Eastaugh N, Walsh V, Chaplin T. Pigment Compendium. Pigment Compend. 2014.

30. Chaplin TD, Clark RJH, Singer BW. Early 20th C Russian painting? Raman identification of modern pigments on a pastel supposedly painted by the renowned artist Natalia Goncharova. J Raman Spectrosc. 2014;45(11-12):1322-5.

31. Saverwyns S. Russian avant-garde or not? A micro-Raman spectroscopy study of sixpaintings attributed to Liubov Popova. J Raman Spectrosc. 2010;41(11):1525-32.

32. Kampasakali E, Varella EA. The Russian avant-garde painting palette: documentary and physicochemical codification of organic colorants. J Cult Herit. 2008;9(1):77-88.

33. Castillejo M, et al. Spectroscopic analysis of pigments and binding media of polychromes by the combination of optical laser-based and vibrational techniques. Appl Spectrosc. 2001;55(8):992-8.

34. Oujja M, Vázquez-Calvo C, Sanz M, De Buergo MÁ, Fort R, Castillejo M. Laser-induced fluorescence and FT-Raman spectroscopy for characterizing patinas on stone substrates. Anal Bioanal Chem. 2012;402(4):1433-41.

35. Bozlee BJ, Misra AK, Sharma SK, Ingram M. Remote Raman and fluorescence studies of mineral samples. Spectrochim Acta Part A Mol Biomol Spectrosc. 2005;61(10):2342-8.

36. Bradley AT, et al. Mineralogy and astrobiology detection using laser remote sensing instrument. Appl Opt. 2015;54(25):7598.

37. Osticioli I, et al. A new compact instrument for Raman, laser-induced breakdown, and laser-induced fluorescence spectroscopy of works of art and their constituent materials. Rev Sci Instrum. 2009:80(7):076109.

38. Sanz M, Oujja M, Castillejo M, Detalle V, Carrasco E, Martínez-Hernández A. Analysis of heritage stones and model wall paintings by pulsed laser excitation of Raman, laser-induced fluorescence and laser-induced breakdown spectroscopy signals with a hybrid system. J Cult Herit. 2018;32:1-8.

39. Anglos $D$, Georgiou S, Fotakis C. Lasers in the analysis of cultural heritage materials. J Nano Res. 2009;8:47-60.

\section{Submit your manuscript to a SpringerOpen ${ }^{\circ}$ journal and benefit from:}

- Convenient online submission

- Rigorous peer review

- Open access: articles freely available online

- High visibility within the field

- Retaining the copyright to your article

Submit your next manuscript at $\boldsymbol{\nabla}$ springeropen.com 